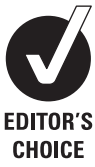

CHOICE

\title{
Management of cancer-associated thrombosis in people with advanced disease
}

\author{
Simon Noble, ${ }^{1}$ Miriam J Johnson²
}

'Department of Palliative Medicine, Cardiff University, Newport, UK

2Department of Health Sciences, Hull York Medical School, Scarborough, UK

\section{Correspondence to}

Dr Simon Noble, Department of Palliative Medicine, Cardiff University, Newport, UK; Simon.noble@wales.nhs.uk

Received 4 November 2011 Accepted 21 January 2012 Published Online First 29 February 2012
The management of venous thromboembolism in the cancer population is clearly established. Low molecular weight heparin has a greater efficacy than warfarin in the treatment of cancer-associated thrombosis and is recommended as the preferred therapy. However, the evidence informing these recommendations excluded patients with poor prognosis or performance status, thrombocytopenia, bleeding or brain metastases. Furthermore, there is limited data on the management of venous thromboembolism resistant to anticoagulation, a phenomenon frequently encountered in the advanced cancer population. This paper will review the management of cancer-associated thrombosis with a particular focus on challenging clinical situations faced by palliative care teams looking after patients with advanced disease.

\section{INTRODUCTION}

The association between cancer and venous thromboembolism (VTE) is widely recognised. People with cancer have a higher risk of developing deep vein thrombosis (DVT) and pulmonary embolus (PE) than those without cancer due to secretion of cancer related procoagulants, which increase with advancing disease and therapeutic interventions including surgery and chemotherapy. ${ }^{1-5}$ Cancer-associated thrombosis has a significant negative impact on survival. ${ }^{67}$

Up to $15 \%$ of cancer patients are estimated to develop clinically apparent VTE, although this is likely to be an underestimate of the problem. ${ }^{14}$ The prevalence of both symptomatic and undiagnosed VTE in advanced disease is thought to be as high as $52 \%$, in keeping with postmortem studies $^{8-10}$ and although the clinical relevance of unsuspected or incidental VTE (typically discovered on staging CT scans) is not fully understood, recent work suggests that cancer patients diagnosed with and treated for incidental PE have similar recurrent VTE and survival to those who had symptomatic PE. 1112

Most participants in the clinical studies that inform the evidence base for the management of cancer-associated VTE were relatively fit ambulant patients (Eastern Cooperative Oncology Group (ECOG) 0-2). Therefore applying treatment guidelines to those with poorer performance status, such as the majority of those cared for in palliative care settings can be difficult. This article reviews the current evidence for managing secondary prevention of cancer-associated thrombosis. In addition it will focus on challenging clinical problems where there is no current evidence to inform practice.

\section{SCOPE OF THE PROBLEM}

Management of patients with VTE and advanced cancer is challenging for several reasons. First, there is an increased risk of bleeding and recurrent VTE with anticoagulation, particularly with vitamin K antagonists (VKA) compared with non-cancer patients. ${ }^{13-24}$ As the cancer progresses, so too does the risk of VTE and bleeding. ${ }^{25}$ Several factors contribute to this: decreased patient mobility, compression of venous return by tumour masses and the presence of highly vascular ulcerating lesions. Furthermore, worsening disseminated intravascular coagulation will result in increasing activation of the coagulation cascade and a tendency to bleed. Thrombocytopenia, due to marrow invasion or as a complication of treatment, may further increase the risk of bleeding.

Second, the advanced cancer population is difficult to define since the term 'advanced cancer patient' refers to a highly heterogeneous group of differing histology, performance status, metastatic burden, mobility and prognosis. These physical variables will affect patients' thombogenicity and bleeding risks. The direction of patient care will often change to a focus on quality of life rather than quantity. It is vitally important to involve patients in their own management plans, particularly at this stage of their lives. Their wishes will be influenced by their current goals and fears in addition to the symptom burden of their disease. For some, a relatively minor insult such as a small PE or minor DVT may have a disproportionately large impact on quality of life while others may consider the potential consequences of treating their VTE to exceed the symptoms.

\section{TREATMENT OF SYMIPTOMITIC VTE}

\section{To treat or not to treat?}

Studies informing the management of cancer-associated thrombosis have all been conducted under the assumption that the patient should receive anticoagulation, with the research question focusing on the choice of anticoagulant to be used. Even in the cancer population, where anticoagulation carries additional hazards, for the majority of patients the benefits of treating symptomatic VTE far outweigh the risks. However, in the palliative care setting the clinician may encounter situations where treating the VTE could be inappropriate or a patient already on anticoagulation should have it discontinued.

There is no evidence to guide these decisionmaking processes and so we would suggest a pragmatic approach. As discussed before, the progression of malignant disease brings with it increasing bleeding complications and the 


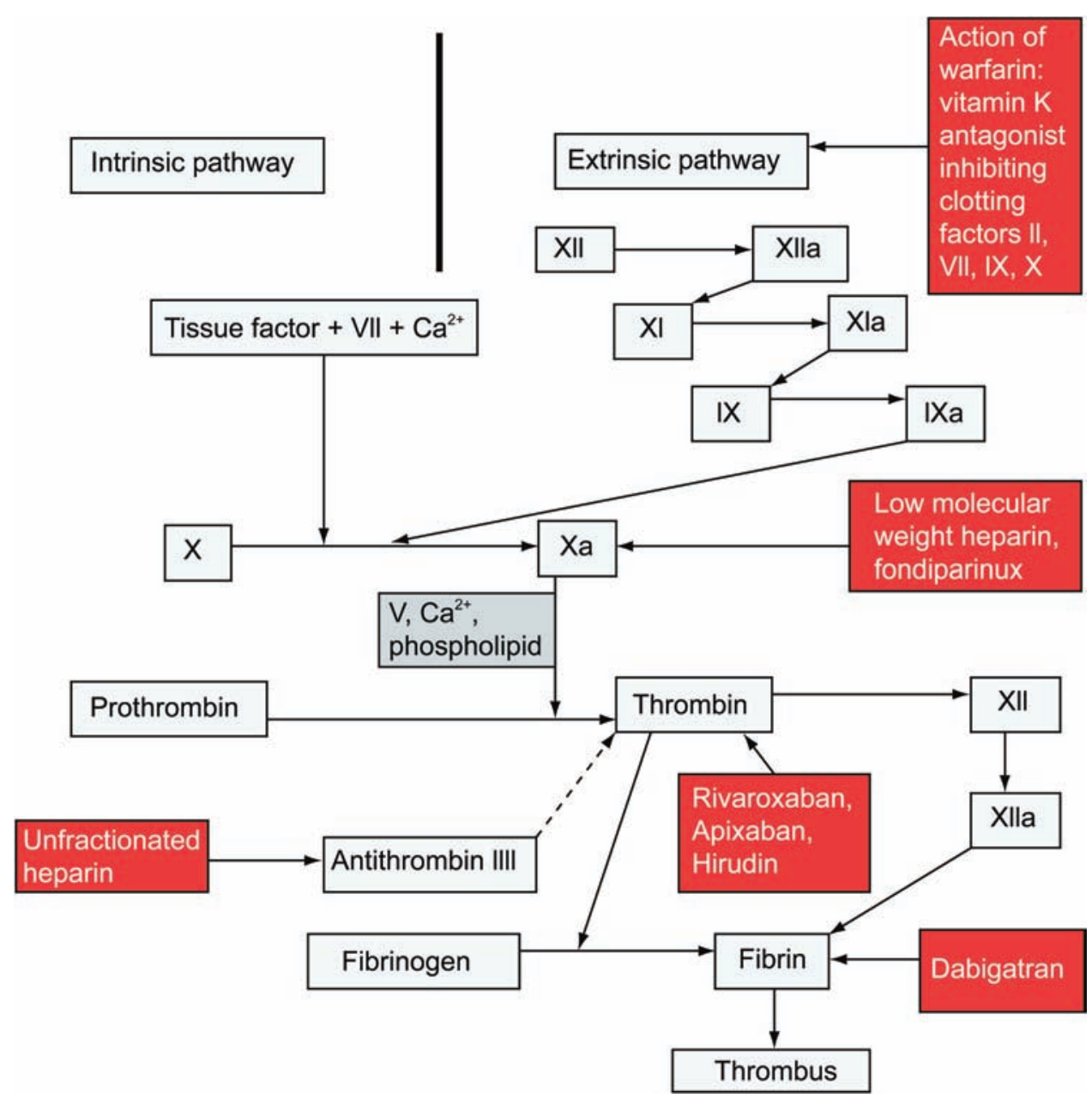

Figure 1 Simplified coagulation cascade illustrating site of action of anticoagulants

potential hazards of anticoagulation should not be underestimated. Long-term anticoagulation in the absence of objective radiological confirmation seems counter intuitive and if investigation of a suspected thrombotic event is considered inappropriate, then arguably so is anticoagulation. Similarly, it would be inappropriate to investigate a suspected thrombus if there were no intention to treat the suspected VTE.

If a patient is near the end of life, with anticipated deterioration any symptoms attributable to VTE could be managed with appropriate end of life medications. For patients who are not imminently dying, anticoagulation may be useful in relieving symptoms attributable to VTE.

\section{Anticoagulation strategies}

The anticoagulants that are commonly used in symptomatic VTE are illustrated in figure 1, which identifies their sites of action within the coagulation cascade. The treatment for VTE in the general non-cancer population consists of 3-6 months anticoagulation with warfarin after initial anticoagulation with weight-adjusted low molecular weight heparin (LMWH). ${ }^{24}$ The use of VKAs such as warfarin in the cancer population, however, is associated with bleeding rates as high as $21.6 \%{ }^{23} 25$ and the initial warfarin loading in advanced cancer patients has reported international normalised ratios (INRs) greater than 4.5 in over $60 \%$ of patients and greater than 8 in $30 \% .{ }^{26}$ Maintaining a stable INR is similarly difficult due to poor nutritional status, liver metastases, variable oral intake/drug absorption and drug-drug interactions.

In addition to an increased risk of bleeding, cancer patients are more likely to develop further thrombotic events on VKAs than non-cancer patients. Studies have shown up $27 \%$ of cancer patients receiving therapeutic VKA will develop secondary VTE. ${ }^{25}$ Although several trials have studied long-term therapy for VTE with oral anticoagulant versus weight-adjusted LMWH, only three have looked at patients with cancer. ${ }^{19} 2122$

Meyer and colleagues randomised patients with cancer and VTE to 3 months of treatment with either LMWH enoxaparin $(1 \mathrm{mg} / \mathrm{kg})$ or warfarin. ${ }^{22}$ The composite outcome of major bleeding and recurrent VTE was observed in 15 out of $71(21.1 \%)$ patients receiving warfarin, compared with 7 of the $67(10.5 \%)$ receiving LMWH ( $p=0.09)$. The long-term innohep treatment evaluation trial randomised 200 patients with acute VTE and cancer to receive either unfractionated heparin (UFH) followed by warfarin for 84 days at a targeted INR of 2.5 or the LMWH tinzaparin (175 IU/kg) for 85 days. ${ }^{19}$ The rate of recurrent VTE at 3 months was $6 \%$ in the LMWH group compared with $10 \%$ in the warfarin group and at 1 year $7 \%$ and $16 \%$ respectively $(p=0.044)$.

The CLOT trial, was a large multicentre study comparing treatment with dalteparin (200 IU/kg for 1 month, then $150 \mathrm{IU} / \mathrm{kg}$ for 5 months) with oral anticoagulant therapy in patients with active cancer presenting with acute VTE. ${ }^{21}$ Three hundred and thirty-eight patients were enrolled into 
Table 1 Randomised control trials comparing oral vitamin $\mathrm{K}$ antagonists with low molecular weight heparin

\begin{tabular}{|c|c|c|c|}
\hline $\begin{array}{l}\text { Recurrent venous } \\
\text { thromboembolism, Warfarin:LWMH }\end{array}$ & $\begin{array}{l}\text { (Calculated from combined endpoint } \\
\text { minus bleeding) } 3 / 75(4 \%): 2 / 71 \text { (2.8\%) }\end{array}$ & $53 / 336(15.8 \%):$ : 27/336 (8\%) & At 3 months: $10 / 100(10 \%)$ : $6 / 100(6 \%)$ \\
\hline & & & At 1 year: $16 / 100(16 \%)$ : $7 / 100(7 \%)$ \\
\hline Bleeding, Warfarin:LMWH & Major: 12/75 (16\%): 5/71 (7\%) & Major: 12/335 (4\%): 19/335 (6\%) & At 3 months: $24 / 100$ (24\%): $27 / 100(27 \%)$ \\
\hline \multirow[t]{3}{*}{ Advanced cancer indices } & Metastatic disease: $52 \%$ & $\begin{array}{l}\text { Performance status } 3,4 \text { and } \\
\text { Weight }<40 \mathrm{~kg} \text { excluded }\end{array}$ & Excluded if $<3$ months prognosis \\
\hline & & $<3$ month prognosis excluded & \\
\hline & Excluded if $<3$ month prognosis & Metastatic disease $67 \%$ & Metastatic disease: warfarin $36 \%$; LMWH 47\% \\
\hline LMWH regime & Enoxaparin $(1.5 \mathrm{mg} / \mathrm{kg})$ & $\begin{array}{l}\text { Dalteparin } 200 \mathrm{IU} / \mathrm{kg} \text { for } 1 \text { month } \\
\text { then } 150 \mathrm{IU} \text { for } 5 \text { months }\end{array}$ & Tinzaparin $(175 \mathrm{IU} / \mathrm{kg})$ \\
\hline Study & Meyer 22 & Lee $^{21}$ & Hull ${ }^{19}$ \\
\hline
\end{tabular}

each arm and were well matched for gender, age, outpatient treatment and performance status. Twenty-seven patients in the LMWH group experienced recurrent VTE compared with 53 in the oral anticoagulant group. Patients receiving long-term LMWH treatment had a significantly lower cumulative risk of recurrent VTE at 6 months than those who received long-term oral anticoagulant therapy $(8.8 \%$ vs $17.4 \% ; 52 \%$ risk reduction; $\mathrm{p}=0.0017)$. Major bleeding was seen in $19 / 336(5.6 \%)$ patients receiving LMWH compared with $12 / 336(3.6 \%)$ in the oral anticoagulant group which was statistically non-significant $(\mathrm{p}=0.27)$. Corresponding data for any bleeding were $13.6 \%$ and $18.5 \%$, respectively $(p=0.09)$. In view of these data, LMWH has been recommended for long-term anticoagulation in cancer patients in clinical guidelines. ${ }^{27-30}$

When considering whether these studies are applicable to those with advanced cancer one must consider the patient exclusion criteria. These are summarised in table 1. All studies excluded those with estimated life expectancy $<3$ months, brain metastases, thrombocytopenia less than $75000 / \mathrm{mm}^{3}$ and active bleeding. Poor performance status was also an exclusion criterion with one study excluding those of ECOG scores $3-4 .{ }^{22}$ However, over $40 \%$ of patients in each study were classed as having metastatic disease or were no longer receiving active treatments for their cancer suggesting the evidence to be applicable to the majority of patients with metastatic disease. Furthermore, a systematic review and meta analysis conducted by an Association for Palliative Medicine of Great Britain and Ireland task group recommended LMWH as the anticoagulant of choice in patients with advanced malignancy. ${ }^{31}$ There is emerging published literature reporting experience of long-term LMWH use in patients with an average median survival of approximately 3 months and included patients with brain metastases; patients who would have been excluded from the above studies. ${ }^{32} 33$

Although LMWH administration requires a daily subcutaneous injection, findings from qualitative research suggest that patients find it an acceptable intervention. ${ }^{34}$ However, it must be noted that most patients participating in the interview study had only been on LMWH for a month, we do not know how they might feel after several months. In addition, these patients had prior poor experience of warfarin since LMWH was not standard practice at the time of the study. Many patients found it preferable to use warfarin since maintenance of a stable INR was reported to require frequent monitoring and these repeated blood tests had a negative impact on quality of life. The health economic implications of using LMWH in advanced cancer patients need to be considered and some services have suggested the cost of LMWH to be prohibitive. ${ }^{35}$
The majority of patients or carers can be taught to administer the drug, negating the potential additional cost of community nursing involvement. ${ }^{32}$

\section{Recurrent VTE despite use of weight-adjusted LMWH}

Occasionally, a cancer patient will have VTE resistant to LMWH because of subtherapeutic levels of the drug or tumour driven direct thrombin activation which will bypass the factor Xa, thus rendering LMWH ineffective. Recurrence of VTE despite therapeutic anticoagulation with LMWH confers a poor prognosis and published data on its management is limited. The focus of management should be on reducing the symptomatic burden without introducing new symptoms from changing therapeutic approaches or complications of therapies.

Possible approaches include increasing the dose of $\mathrm{LMWH}$, if sub-therapeutic, changing to twice-daily injections to minimise trough levels, or switching to intravenous or subcutaneous UFH, where direct thrombin activation is suspected or rapid reversal in a highly thrombotic state prior to surgery is needed.

\section{Initial management}

In the CLOT study, the LWMH dose was reduced to $75 \%$ after 1 month. ${ }^{21}$ Therefore the initial management of recurrent VTE should be to ensure that, if the dose has been previously reduced, full weight adjusted dose LMWH is reinstated. If this does not work, there is little in the published literature to guide the clinician. A case series of 47 cancer patients with recurrent VTE despite full weight-adjusted LMWH describes resolution of VTE attributable symptoms within following an empirical increase in LMWH dose by $25 \% .{ }^{36}$ However, there is insufficient evidence to recommend this routinely. The utility of anti-Xa level monitoring in this situation is unclear. To date there are no studies supporting the use of anti-Xa levels to guide LMWH dosing in the cancer patient and published data is limited to a single case report. ${ }^{37}$ Further management requires liaison with haematology colleagues for guidance and it is likely that treatment plans will be developed on an individual patient basis. The role of anti-Xa levels in the dosing of LMWH in cancer-associated thrombosis needs further research.

\section{Direct thrombin activation}

Assuming LMWH levels are therapeutic, it is possible that direct thrombin activation is the underlying driver of recurrent VTE and a change to UFH may be warranted. The new oral direct thrombin inhibitors may have a future role to play in the 
management of this challenging patient group. However, it is unclear yet whether the theoretical benefit of direct thrombin inhibition will bring with it an increased risk of bleeding. The role of new oral anticoagulants including direct thrombin and factor Xa inhibitors is discussed later in this article.

\section{VTE in thrombocytopenia}

Thrombocytopenia increases the risk of bleeding in cancer patients and is elevated further by anticoagulation. LMWH has not been formally evaluated in patients with a platelet count lower than $75000 / \mathrm{mm}^{3}$ and thus guidelines do not recommend LMWH in this situation. However, in patients with symptomatic proximal DVT or PE, options may be limited and the risks of anticoagulation with LMWH must be weighed against the consequences of choosing not to anticoagulate. There is some clinical experience of LMWH anticoagulation in cancer patients with platelet counts above $50000 / \mathrm{mm}^{3}$ and from the authors experience, LMWH has been used in extreme cases when platelets were as low as $25000 / \mathrm{mm}^{3} .{ }^{26}$ Monreal et al have described the use of dose reduction in people with thrombocytopenia, using doses as low as 2500 IU dalteparin in those with thrombocytopenia $<10000 / \mathrm{mm}^{3}$. 38 Those with thrombocytopenia did not have a higher bleeding complication rate than those without.

\section{VTE in patients with bleeding}

There is no published evidence to guide the clinician in this particularly challenging situation and management should be tailored to the individual after full discussion of the risks of each treatment option. ${ }^{26}$ A sensible first step is to try and stop the bleeding if possible. If this is not possible, then nuisance bleeding such as mild epistaxis or haemoptysis should not, in our opinion, prevent anticoagulation in patients with symptomatic VTE in whom the risk of clinically significant recurrence is high. If the bleeding is from a visible, easily monitored source which is unlikely to be life threatening then full anticoagulation should commence (or be continued) with careful review. If the bleeding is due to mucosal involvement by tumour, such as bowel, duodenal, bladder or vaginal disease a more cautious approach of reduced or even prophylactic doses should be used and the patient monitored closely. The haemostatic properties of a palliative radiotherapy should be considered as it is well tolerated and embolisation in carefully selected patients may be considered. ${ }^{39}$ If the haemoglobin remains stable and bleeding does not worsen, the dose can be slowly titrated up. A twice-daily LMWH regime may smooth out peaks in anticoagulation level. If there is active bleeding of a more serious and potentially life threatening nature (intracranial, retroperitoneal or upper gastrointestinal bleeding), then anticoagulation is contraindicated.

\section{USE OF VENA CAVAL FILTERS}

There is no evidence that insertion of a vena caval filter improves outcome for cancer patients with VTE who are bleeding and published studies are too small for useful conclusions. ${ }^{40-45}$ The evidence in the advanced cancer population is even weaker and given inferior vena cava (IVC) filters do not reduce symptoms of VTE or improve survival, the use of these devices are limited in the palliative setting.

However, for patients with contraindications to anticoagulation, or who continue to embolise despite therapeutic anticoagulation, clinicians may consider there to be few, if any, alternative options and carefully selected, individuals may gain net-benefit. It is important to note that filter insertion does not suppress the underlying hypercoagulable state, and may even aggravate it. In addition, there is a concern that fatal PE might still result from thrombus formation in the vena cava proximal to the filter.

\section{DURATION OF THERAPY}

The length of treatment with an anticoagulant for the secondary prevention of recurrent VTE has not been studied in cancer patients of any stage disease. However, based on the consensus that patients with ongoing or irreversible risk factors for VTE need an extended duration of anticoagulant therapy, a minimum of 3-6 months of treatment is prescribed. Beyond this period, 'indefinite' therapy is traditionally recommended in patients with metastatic disease because their risk of recurrent VTE is high. In a recent study in 62 patients with advanced, metastatic cancer and VTE, three of the seven patients who stopped LMWH after 6 months of treatment developed symptomatic, recurrent thrombosis. ${ }^{32}$ This has recently been recognised as a priority for research. ${ }^{46}$

\section{FUTURE CONSIDERATIONS}

Therapeutic advances in cancer management have resulted in people living longer with metastatic disease. VTE will continue to be a challenge in people with advanced cancer because the evidence base is extrapolated from healthier populations using outcome measures which may be less applicable to patients of poor performance status and prognosis. Clinical research in this group has always been fraught with difficulty and robust studies within representative populations will require large-scale collaboration between research teams if sufficient numbers are to be recruited. Likewise, palliative care practice will only change if the evidence is applicable and the outcome measures are meaningful. ${ }^{47}$ A greater emphasis on patient reported outcome measures, the symptomatic burden of VTE and its therapies as well as over all quality of life assessments are needed.

Finally, release of new oral anticoagulants including direct $\mathrm{Xa}$ inhibitors (rivaroxaban, apixaban) and direct thrombin inhibitors (dabigatran etexilate) are potentially exciting developments in the prevention and treatment of VTE in the palliative care setting. Both dabigatran etexilate and rivaroxaban have been evaluated in the treatment of DVT and PE showing non-inferiority to warfarin with respect to recurrent VTE and bleeding. ${ }^{48}{ }^{49}$ However, the use of dabigatran and rivaroxaban has been cautioned in high-risk groups such as cancer patients until studies with representative numbers of cancer patients are available. ${ }^{50} 51$ Only $5 \%$ and $6.8 \%$ of patients, respectively, had cancer and non-inferiority of these agents in this setting, compared with the current gold standard (LMWH) that is yet to be demonstrated. However, until their evaluation in other medical populations has been concluded, their potential role in the advanced cancer population remains theoretical.

Contributors SN and MJJ both conceived the idea for the paper and co-authored the paper together.

\section{Competing interests None.}

Provenance and peer review Not commissioned; externally peer reviewed.

\section{REFERENCES}

1. Bick RL. Alterations of hemostasis associated with malignancy: etiology, pathophysiology, diagnosis and management. Semin Thromb Hemost 1978;5:1-26.

2. Johnson MJ, Walker ID, Sproule MW, et al. Abnormal coagulation and deep venous thrombosis in patients with advanced cancer. Clin Lab Haematol 1999;21:51-54. 
3. Kakkar AK, DeRuvo N, Chinswangwatanakul V, et al. Extrinsic-pathway activation in cancer with high factor VIla and tissue factor. Lancet 1995;346:1004-1005.

4. Levitan N, Dowlati A, Remick SC, et al. Rates of initial and recurrent thromboembolic disease among patients with malignancy versus those without malignancy. Risk analysis using Medicare claims data. Medicine (Baltimore) 1999;78:285-291.

5. Sampson MT, Kakkar AK. Coagulation proteases and human cancer. Biochem Soc Trans 2002;30:201-207.

6. Mandalà $\mathbf{M}$, Reni $\mathrm{M}$, Cascinu $\mathrm{S}$, et al. Venous thromboembolism predicts poor prognosis in irresectable pancreatic cancer patients. Ann Oncol 2007; 18:1660-1665.

7. Alcalay A, Wun T, Khatri V, et al. Venous thromboembolism in patients with colorectal cancer: incidence and effect on survival. J Clin Oncol 2006;24:1112-1118.

8. Ambrus JL, Ambrus CM, Mink IB, et al. Causes of death in cancer patients. J Med 1975;6:61-64

9. Johnson MJ, Sproule MW, Paul J. The prevalence and associated variables of deep venous thrombosis in patients with advanced cancer. Clin Oncol (R Coll Radiol) 1999;11:105-110.

10. Sproul EE. Carcinoma and venous thrombosis: the frequency of association of carcinoma in the body or tail of the pancreas with multiple venous thrombosis. Am J Cancer 1938;34:566-585.

11. Abdel-Razeq HN, Mansour AH, Ismael YM. Incidental pulmonary embolism in cancer patients: clinical characteristics and outcome-a comprehensive cancer center experience. Vasc Health Risk Manag 2011;7:153-158.

12. den Exter PL, Hooijer J, Dekkers OM, et al. Risk of recurrent venous thromboembolism and mortality in patients with cancer incidentally diagnosed with pulmonary embolism: a comparison with symptomatic patients. J Clin Oncol 2011;29:2405-2409.

13. Chan A, Woodruff RK. Complications and failure of anticoagulation therapy in the treatment of venous thromboembolism in patients with disseminated malignancy. Aust N Z J Med 1992;22:119-122.

14. Clarke-Pearson DL, Synan IS, Creasman WT. Anticoagulation therapy for venous thromboembolism in patients with gynecologic malignancy. Am J Obstet Gynecol 1983:147:369-375.

15. Debourdeau P, Meyer G, Sayeg H, et al. [Classical anticoagulant treatment of venous thromboembolic disease in cancer patients. Apropos of a retrospective study of 71 patients]. Rev Med Interne 1996;17:207-212.

16. Deitcher SR, Kessler CM, Merli G, et al. Secondary prevention of venous thromboembolic events in patients with active cancer: enoxaparin alone versus initial enoxaparin followed by warfarin for a 180-day period. Clin App/ Thromb Hemost 2006;12:389-396.

17. Gitter MJ, Jaeger TM, Petterson TM, et al. Bleeding and thromboembolism during anticoagulant therapy: a population-based study in Rochester, Minnesota. Mayo Clin Proc 1995;70:725-733.

18. Harrington KJ, Bateman AR, Syrigos KN, et al. Cancer-related thromboembolic disease in patients with solid tumours: a retrospective analysis. Ann Oncol 1997;8:669-673.

19. Hull RD, Pineo GF, Brant RF, et al. Long-term low-molecular-weight heparin versus usual care in proximal-vein thrombosis patients with cancer. Am J Med 2006;119:1062-1072.

20. Krauth D, Holden A, Knapic N, et al. Safety and efficacy of long-term oral anticoagulation in cancer patients. Cancer 1987;59:983-985

21. Lee AY, Levine MN, Baker RI, et al. Low-molecular-weight heparin versus a coumarin for the prevention of recurrent venous thromboembolism in patients with cancer. N Engl J Med 2003;349:146-153.

22. Meyer G, Marjanovic Z, Valcke J, et al. Comparison of low-molecular-weight heparin and warfarin for the secondary prevention of venous thromboembolism in patients with cancer: a randomized controlled study. Arch Intern Med 2002;162:1729-1735.

23. Moore FD Jr, Osteen RT, Karp DD, et al. Anticoagulants, venous thromboembolism, and the cancer patient. Arch Surg 1981;116:405-407.

24. Paloretti G. A comparison of the safety and efficacy of oral anticoagulation for the treatment of venous thromboembolic disease in patients with or without malignancy. Legnani C, Lee A, eds. Thromb Haemost 2000;84:805-810.

25. Prandoni $\mathbf{P}$, Lensing AW, Piccioli A, et al. Recurrent venous thromboembolism and bleeding complications during anticoagulant treatment in patients with cancer and venous thrombosis. Blood 2002:100:3484-3488.

26. Lee A. Treatment and secondary prevention of venous thromboembolism in advanced cancer. In: Noble SIR, Johnson MJ, Lee A, eds. Venous Thromboembolism in Advanced Disease: a clinical guide. First edition. Oxford University Press 2008:91-110.
27. Mandalà $\mathbf{M}$, Falanga $\mathrm{A}$, Piccioli $\mathrm{A}$, et al. Venous thromboembolism and cancer: guidelines of the Italian Association of Medical Oncology (AIOM). Crit Rev Oncol Hematol 2006:59:194-204.

28. Lyman GH, Khorana AA, Falanga A et al. American Society of Clinical Oncology. American Society of Clinical Oncology guideline: Recommendations for Venous Thromboembolism Prophylaxis and Treatment in Patients with Cancer. J Clin Oncol 2007:25:490-505.

29. Segal JB, Streiff MB, Hofmann LV, et al. Management of venous thromboembolism: a systematic review for a practice guideline. Ann Intern Med 2007:146:211-222

30. Kearon C, Kahn SR, Agnelli G, et al. Antithrombotic therapy for venous thromboembolic disease: American College of Chest Physicians Evidence-Based Clinical Practice Guidelines (8 $8^{\text {th }}$ Edition). Chest 2008;133:454S-545S.

31. Noble SI, Shelley MD, Coles B, et al. Management of venous thromboembolism in patients with advanced cancer: a systematic review and meta-analysis. Lancet Oncol 2008; 9:577-584

32. Noble SI, Hood K, Finlay IG. The use of long-term low-molecular weight heparin for the treatment of venous thromboembolism in palliative care patients with advanced cancer: a case series of sixty two patients. Palliat Med 2007;21:473-476.

33. Soto-Cárdenas MJ, Pelayo-García G, Rodríguez-Camacho A, et al. Venous thromboembolism in patients with advanced cancer under palliative care: additional risk factors, primary/secondary prophylaxis and complications observed under normal clinical practice. Palliat Med 2008;22:965-968.

34. Noble SI, Finlay IG. Is long-term low-molecular-weight heparin acceptable to palliative care patients in the treatment of cancer related venous thromboembolism? A qualitative study. Palliat Med 2005;19:197-201.

35. Tassinari D, Santelmo C, Scarpi E, et al. Controversial issues in thromboprophylaxis with low-molecular weight heparins in palliative care. J Pain Symptom Manage 2008;36:e3-e4.

36. Carrier M, Le Gal G, Cho R, et al. Dose escalation of low molecular weight heparin to manage recurrent venous thromboembolic events despite systemic anticoagulation in cancer patients. J Thromb Haemost 2009;7:760-765.

37. Cunningham MS, Gilmore R, O'Donnell D, et al. Management of refractory venous thrombosis associated with malignancy using a supra-therapeutic enoxaparin regimen. Ir J Med Sci 2009;178:339-341.

38. Monreal M, Zacharski L, Jiménez JA, et al. Fixed-dose low-molecular-weight heparin for secondary prevention of venous thromboembolism in patients with disseminated cancer: a prospective cohort study. J Thromb Haemost 2004;2:1311-1315.

39. Biswal BM, Lal P, Rath GK, et al. Hemostatic radiotherapy in carcinoma of the uterine cervix. Int J Gynaecol Obstet 1995;50:281-285

40. Calligaro KD, Bergen WS, Haut MJ, et al. Thromboembolic complications in patients with advanced cancer: anticoagulation versus Greenfield filter placement. Ann Vasc Surg 1991;5:186-189.

41. Cohen JR, Tenenbaum N, Citron M. Greenfield filter as primary therapy for deep venous thrombosis and/or pulmonary embolism in patients with cancer. Surgery 1991:109:12-15

42. Olin JW, Young JR, Graor RA, et al. Treatment of deep vein thrombosis and pulmonary emboli in patients with primary and metastatic brain tumors. Anticoagulants or inferior vena cava filter? Arch Intern Med 1987:147:2177-2179.

43. Walsh DB, Downing S, Nauta R, et al. Metastatic cancer. A relative contraindication to vena cava filter placement. Cancer 1987:59:161-163.

44. Whitney BA, Kerstein MD. Thrombocytopenia and cancer: use of the Kim-Ray Greenfield filter to prevent thromboembolism. South Med J 1987;80:1246-1248.

45. Zerati AE, Wolosker N, Yazbek G, et al. Vena cava filters in cancer patients: experience with 50 patients. Clinics (Sao Paulo) 2005;60:361-366.

46. NIHR Health Technology Assessment Programme. Duration of treatment of venous thromboembolism in malignant disease. HTA no 10/145, 2011.

47. Noble SI, Nelson A, Finlay IG. Factors influencing hospice thromboprophylaxis policy: a qualitative study. Palliat Med 2008;22:808-813.

48. Bauersachs R, Berkowitz SD, Brenner B, et al. Oral rivaroxaban for symptomatic venous thromboembolism. N Engl J Med 2010:363:2499-2510.

49. Teachey DT. Dabigatran versus warfarin for venous thromboembolism. N Eng/ J Med 2010;362:1050; author reply 1050-1050; author reply 1051.

50. Peetz D, Lackner KJ. Dabigatran versus warfarin for venous thromboembolism. N Eng/ J Med 2010;362:1050; author reply 1050-1050; author reply 1051.

51. Romualdi E, Donadini MP, Ageno W. Oral rivaroxaban after symptomatic venous thromboembolism: the continued treatment study (EINSTEIN-Extension study). Expert Rev Cardiovasc Ther 2011;9:841-844 